\title{
Fitness benefits of loss of heterozygosity in Saccharomyces hybrids
}

\author{
Samuel M. Lancaster, ${ }^{1,2}$ Celia Payen,, ${ }^{1,3}$ Caiti Smukowski Heil, ${ }^{1,4}$ \\ and Maitreya J. Dunham ${ }^{1}$ \\ ${ }^{1}$ Department of Genome Sciences, University of Washington, Seattle, Washington 98195, USA
}

\begin{abstract}
With two genomes in the same organism, interspecific hybrids have unique fitness opportunities and costs. In both plants and yeasts, wild, pathogenic, and domesticated hybrids may eliminate portions of one parental genome, a phenomenon known as loss of heterozygosity ( $\mathrm{LOH}$ ). Laboratory evolution of hybrid yeast recapitulates these results, with $\mathrm{LOH}$ occurring in just a few hundred generations of propagation. In this study, we systematically looked for alleles that are beneficial when lost in order to determine how prevalent this mode of adaptation may be and to determine candidate loci that might underlie the benefits of larger-scale chromosome rearrangements. These aims were accomplished by mating Saccharomyces uvarum with the $S$. cerevisiae deletion collection to create hybrids such that each nonessential $S$. cerevisiae allele is deleted. Competitive fitness assays of these pooled, barcoded, hemizygous strains, and accompanying controls, revealed a large number of loci for which $\mathrm{LOH}$ is beneficial. We found that the fitness effects of hemizygosity are dependent on the species context, the selective environment, and the species origin of the deleted allele. Further, we found that hybrids have a wider distribution of fitness consequences versus matched $S$. cerevisiae hemizygous diploids. Our results suggest that LOH can be a successful strategy for adaptation of hybrids to new environments, and we identify candidate loci that drive the chromosomal rearrangements observed in evolution of yeast hybrids.
\end{abstract}

[Supplemental material is available for this article.]

Hybrid organisms are common in nature, particularly in fungi and plants, where an estimated $25 \%$ of plants and $10 \%$ of animals are hybrids (Mallet 2005). Even the human genome is now recognized to contain substantial introgressions-remnants of ancient hybridization-that are thought to be adaptive (Huerta-Sánchez et al. 2014; Dannemann et al. 2016; Gittelman et al. 2016; Racimo et al. 2017). Hybrids have been created via artificial selection in agriculture, industry, and the laboratory. For example, wheat, a pillar of civilization, is a triple hybrid between three grass species (Brenchley et al. 2012). Hybridization and introgression are also abundant in budding yeast (for review, see Morales and Dujon 2012), where hybrids have been found to possess adaptive advantages over their parental species (e.g., Stelkens et al. 2014), show desirable properties as industrial organisms (Mertens et al. 2015; Peris et al. 2017), and contribute to the emergence of fungal pathogens (Morales and Dujon 2012; Pryszcz et al. 2015; Schröder et al. 2016; Mixão and Gabaldón 2018). A whole-genome duplication ancestral to Saccharomyces yeasts-a defining characteristic of the clade-has been recognized as a hybridization event (MarcetHouben and Gabaldón 2015). Since Saccharomyces has relatively weak prezygotic barriers to speciation (Maclean and Greig 2008; Murphy and Zeyl 2012), Saccharomyces is particularly rife with hybridization and includes hybridization between species as distant as 20 million years diverged ( $\sim 80 \%$ amino acid and nucleotide identity), which are capable of intermating (Martini and Martini 1987; Naumova et al. 2005; Dunn and Sherlock 2008; Muller and McCusker 2009; Libkind et al. 2011; Nguyen et al. 2011;

Present addresses: ${ }^{2}$ Department of Genetics, Stanford University, Stanford, CA 94304, USA; ${ }^{3}$ Industrial Biosciences DuPont, Wilmington, DE 19803, USA; ${ }^{4}$ Biological Sciences Department, North Carolina State University, Raleigh, NC 27695, USA Corresponding author: maitreya@uw.edu

Article published online before print. Article, supplemental material, and publication date are at http://www.genome.org/cgi/doi/10.1101/gr.245605.118.
Almeida et al. 2014; Pérez-Través et al. 2014). Two common yeasts that originated as hybrids between $S$. cerevisiae and cryotolerant species have even received designation as hybrid species: the wine yeast $S$. bayanus, a triple hybrid between $S$. cerevisiae, $S$. uvarum, and S. eubayanus (Gonzalez et al. 2006; Sipiczki 2008); and the lager yeast $S$. pastorianus, which is a hybrid between $S$. cerevisiae and S. eubayanus (Martini and Martini 1987; Dunn and Sherlock 2008; Libkind et al. 2011; Nguyen et al. 2011; Pérez-Través et al. 2014). These species highlight the observation that fermentation environments are particularly rich in hybrids, spanning genera including Saccharomyces, Dekkera, and Pichia (Borneman et al. 2014; Smukowski Heil et al. 2018).

Similar to plant hybrids (for review, see Chester et al. 2010), yeast hybrids can shed large portions of their genomes from one or both species during evolution (Otto and Whitton 2000; Sun and Xu 2009; Chester et al. 2010; Csoma et al. 2010; Louis et al. 2012; Peris et al. 2012; Pryszcz et al. 2015; Chen et al. 2017; Emery et al. 2018). Resolution of the ancestral whole-genome duplication in Saccharomyces involved loss of the majority of duplicated genes, in a process that began shortly after the initial hybridization event (Scannell et al. 2007). These large-scale changes in genome structure and content have been recapitulated in part in the laboratory, demonstrating the rapidity with which these changes can occur and confirming their potential contribution to adaptation. For example, experimental evolution of yeast hybrids under a number of selective conditions found genome rearrangements after only a few hundred generations (KunickaStyczyńska and Rajkowska 2011; Piotrowski et al. 2012; Sanchez

(C) 2019 Lancaster et al. This article is distributed exclusively by Cold Spring Harbor Laboratory Press for the first six months after the full-issue publication date (see http://genome.cshlp.org/site/misc/terms.xhtml). After six months, it is available under a Creative Commons License (Attribution-NonCommercial 4.0 International), as described at http://creativecommons.org/licenses/by$\mathrm{nc} / 4.0 /$. 
et al. 2017; Smukowski Heil et al. 2017). The genome regions affected are dependent on the selective pressure used, and observed events include whole chromosome aneuploidy, both focal and chromosome arm amplifications, translocations, gene fusions, and $\mathrm{LOH}$. Our previous work demonstrated that $\mathrm{LOH}$ can result from selection on one species allele and loss of the other. Using a candidate gene approach, we identified a single gene (PHO84) whose allelic differences explained the majority of the fitness benefits in evolved populations relative to their ancestor. However, additional, as yet unidentified driver genes must exist to fully account for the evolved strains' fitness improvements, and many observed $\mathrm{LOH}$ regions remain unexplained. More broadly, the degree to which LOH is a product of genetic drift versus selection is not yet clear. To further complicate matters, improved fitness caused by LOH could have a number of possible explanations, including selecting for the better species' alleles, uncovering beneficial recessive alleles, and/or resolving hybrid incompatibilities. Studying LOH in hybrid yeast allows a systematic approach that facilitates insights into these phenomena and provides a foundation to guide further investigation into hybrid biology in other, less tractable contexts.

Such systematic approaches have been made possible via the creation of genome-scale deletion collections, including a nearcomprehensive set of diploid $S$. cerevisiae strains hemizygous for every gene (Giaever et al. 2002; Deutschbauer and Davis 2005). These strains were created such that each carries a unique DNA barcode, facilitating pooled assays for competitive growth in a variety of conditions. Many studies have illustrated that heterozygous deletions can cause fitness defects ("haploinsufficiency"), and a smaller number have also found fitness increases, or haploproficiency (Delneri et al. 2008; Pir et al. 2012; Ohnuki and Ohya 2018; Weiss et al. 2018). Previously, in order to determine driver mutations, our lab identified haploinsufficient and haploproficient loci in the deletion collection in environments matching laboratory evolution studies (Payen et al. 2016). However, since these loci were identified in $S$. cerevisiae diploids, the degree to which they explain the prevalence of, and genetic drivers for, hemizygosity in hybrids is unclear. There is reason to believe that loci important for hybrid adaptation likely differ from those important in purebred diploids. For example, Herbst et al. (2017) found that in $S$. paradoxus $\times S$. cerevisiae hybrids, hundreds of allelic deletions affect the growth rate of hybrids but not of $S$. cerevisiae diploids.

In order to understand hybrid $\mathrm{LOH}$, in this study we utilized two divergent species: $S$. cerevisiae and $S$. uvarum. We previously evolved hybrids and diploids of these species in nutrient-limited chemostat culture (Gresham et al. 2008; Sanchez et al. 2017; Smukowski Heil et al. 2017, 2019). We created thousands of S. cerevisiae $\times S$. uvarum hybrid yeast strains by mating the $S$. cerevisiae nonessential deletion collection to WT $S$. uvarum. These collections of hybrid yeast, along with control populations, were assayed for competitive fitness in three nutrient-limited environments matched to our previous evolution conditions. Our study demonstrates that hybrids offer a unique fitness landscape with potentially more beneficial mutations, which may contribute to their ability to adapt, and it provides attractive candidate genes for future study.

\section{Results}

We sought to discover the genome-wide fitness effects of hemizygosity in hybrid Saccharomyces in three nutrient-limited conditions that correspond with those previously used for experimental evolution. To this end, we mated $S$. uvarum to the $S$. cerevisiae haploid deletion collection creating thousands of hybrid yeast strains, each with a $S$. cerevisiae allele deleted. For comparison, we used a matched collection of $S$. cerevisiae hemizygous deletion strains. Additionally, we created control collections of thousands of WT S. cerevisiae and WT hybrid strains that contain unique DNA barcodes but are otherwise isogenic. (All collections are described in Table 1.) These control libraries allowed us to empirically measure technical and biological variation in our strain construction, growth, and sequencing pipeline. All strains were assayed for relative fitness via pooled competitive growth for 25 generations in glucose-, phosphate-, and sulfate-limited chemostat culture followed by barcode sequencing. The barcode counts track strain abundance over time, allowing us to derive competitive fitness scores (see Methods; Supplemental Table S1). Each experiment was performed in biological replicate (Supplemental Fig. S1). We confirmed that this pooled approach accurately reflects strain fitness by comparing the results to pairwise competitions of individual deletion strains versus a GFP-marked WT competitor (Supplemental Fig. S2; Supplemental Table S2).

\section{Fitness effects of hemizygosity in S. cerevisiae diploids}

Both collections of control WT strains have narrow fitness distributions around neutrality, with $98 \%$ of the $S$. cerevisiae controls falling between fitness values of 0.047 and -0.040 and $98 \%$ of hybrid controls between 0.046 and -0.032 across all experiments (Supplemental Fig. S3). We used these empirical 1\% cutoffs to determine significant increases and decreases in fitness of the deletion strains. Out of a total of 6003 possible deletion strains, we identified 4806 strains by barcode sequencing in the glucose-limited competition, 4855 strains in phosphate limitation, and 4901 strains in sulfate limitation. Compared to the WT control distribution, hemizygous gene deletions in S. cerevisiae caused a broader distribution of fitness effects. The null expectation for $1 \%$ cutoffs would be 48,49 , and 49 outliers in each direction for glucose, phosphate, and sulfate limitations, respectively. We observe significantly more deletion strains with fitness values beyond our cutoffs in several

Table 1. Strain collections and data sets used in this study

\begin{tabular}{ll}
\hline Collection & \multicolumn{1}{c}{ Description } \\
\hline $\begin{array}{l}\text { S. cerevisiae Hemizygous Deletion Collection } \\
\text { ("purebred" collection) }\end{array}$ & $\begin{array}{l}\text { S. cerevisiae hemizygous deletion collection from Giaever et al. (2002); fitness data from Payen et al. } \\
(2016)\end{array}$ \\
$\begin{array}{l}\text { Hybrid Hemizygous Deletion Collection } \\
\text { S. cerevisiae Barcoded Control Library }\end{array}$ & $\begin{array}{l}\text { S. cerevisiae haploid deletion collection from Giaever et al. (2002) mated to WT S. uvarum } \\
\text { Collon of barcoded WT haploid S. cerevisiae strains from Yan et al. (2008) mated to WT, } \\
\text { Collection of barcoded WT haploid S. cerevisiae strains from Yan et al. (2008) mated to } \\
\text { Wybrid Barcoded Control Library }\end{array}$ \\
WT S. uvarum
\end{tabular}

\section{Genome Research}

www.genome.org 
conditions: 308 haploinsufficient genes and 64 haploproficient genes in glucose limitation $\left(P<2.2 \times 10^{-16}, P=0.19\right) ; 163$ and 5 in phosphate limitation $\left(P=4.3 \times 10^{-15}, P=4.4 \times 10^{-9}\right.$ fewer $)$; and 58 and 113 in sulfate limitation $\left(P=0.44, P=6 \times 10^{-7}\right.$; comparison of two population proportions performed in R [R Core Team 2017], with Yates continuity correction). Thus, we conclude that, in the $S$. cerevisiae hemizygous collection, there are more deletions that cause extreme fitness effects than we would expect by chance, consistent with our previous results (Payen et al. 2016).

\section{Fitness effects of $\mathrm{LOH}$ are more extreme in hybrids}

We applied the same analysis as above to the hybrid deletion strains. Out of 4828 possible deletion strains (a lower number than above because only nonessential $S$. cerevisiae gene deletions can be used), we identified 3195 deletion strains in sulfate limitation, 3179 in phosphate limitation, and 2955 in glucose limitation. Under the null expectation set by our $1 \%$ cutoff, we would expect 32 outliers in each direction in sulfate-limited culture, 32 in phosphate-limited, and 30 in glucose-limited. However, in the hybrids we observed 308 haploinsufficient genes and 63 haploproficient genes in glucose limitation $\left(P<2.2 \times 10^{-16}, P=0.0008\right) ; 919$ and 453 in phosphate limitation $\left(P<2.2 \times 10^{-16}, P<2.2 \times 10^{-16}\right)$; and 216 and 17 in sulfate limitation $\left(P<2.2 \times 10^{-16}, P>0.05\right.$; comparison of two population proportions performed in $\mathrm{R}$, with Yates continuity correction). The differences between nutrient limitations are illustrated in the different shapes of the distributions (Supplemental Fig. S3). In phosphate limitation, the highest fitness strains had risen to an abundance $>1.5 \%$ of the population by the final time point, over two orders of magnitude above their initial frequency.

Hybrid deletion mutants had a significantly broader range of fitness values than deletions of the same loci in the $S$. cerevisiae diploid context (Levene test, $P=1.9 \times 10^{-12}, P<2.2 \times 10^{-16}, P=8.6 \times$ $10^{-6}$, for glucose, phosphate, and sulfate limitations, respectively) (Supplemental Fig. S3), suggesting loss of an allele in hybrids leads to more extreme fitness outcomes, in both directions (Supplemental Fig. S4). There were a few notable features in the distribution, including a bulge of strains in the haploinsufficient distribution of sulfate limitation. The only Gene Ontology (GO) term significant in this portion was "RNA metabolic process" $(P=0.008$, Bonferroni corrected), suggesting that genes associated with the process are more sensitive to deletion in this environment and genetic context, though for as yet undetermined reasons.

We next compared genes that had significant fitness effects in hybrids to those in the $S$. cerevisiae diploids. Although there were some genes that were consistent between genetic backgrounds, correlation was low, and some gene deletions even had inverse effects (Fig. 1). Consistent with these findings, the genes identified in the hybrid and S. cerevisiae diploid data sets had different GO enrichments (Supplemental Tables S3, S4). Individual strains showed several different patterns in this experiment. Strains in blue showed a consistent increase in fitness, regardless of whether the deletion was in the hybrid or purebred. Strains in red showed a consistent decrease. Green strains had pleotropic effects, where the genotype was beneficial in one condition and detrimental in another. Purple strains showed a change in fitness in only one genetic context, and black strains showed minimal changes in fitness in either genetic context (Fig. 1).

Highlighting the effect of genetic background, many of the haploinsufficient alleles in the purebred genetic context are alleviated in the hybrid context, defined as an increase in fitness of at

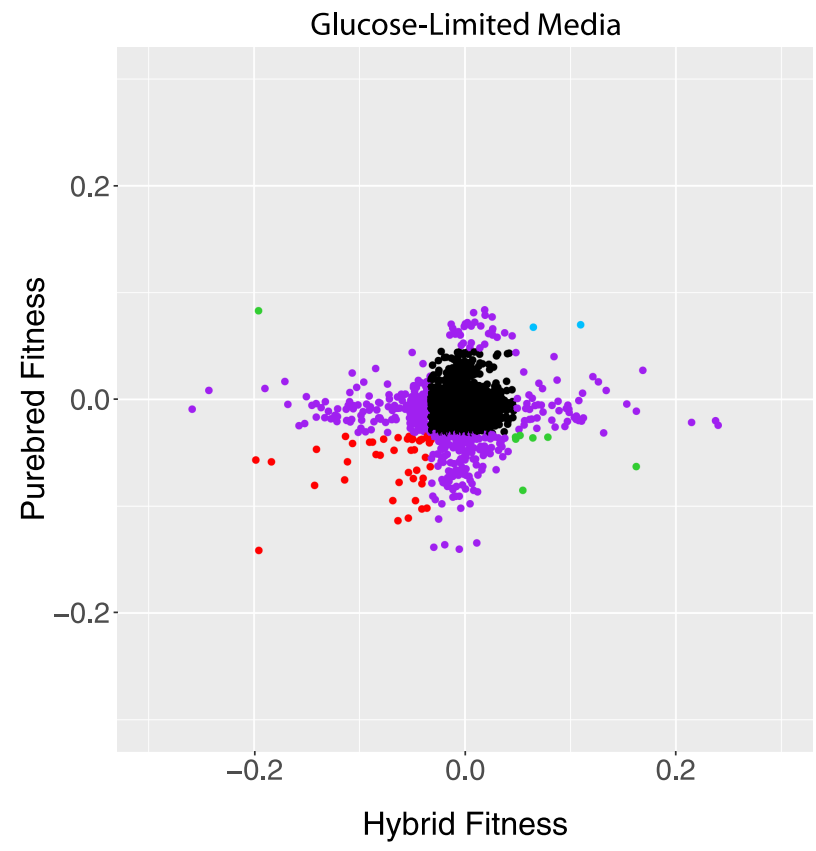

Figure 1. Scatter plot of fitness values for hybrid and diploid S. cerevisiae strains hemizygous for deletion mutations, measured in glucose limitation (comparisons in other nutrient limitations can be found in Supplemental Fig. S4). Black strains fall inside the $1 \%$ cutoff in both axes, purple strains fall outside the $1 \%$ cutoff in just one axis, and the other colors fall outside of the cutoffs in both axes. Data from Supplemental Table S1. $R^{2}=0.00$.

least 0.04 , which is the $95 \%$ cutoff for the purebred collection. In glucose, phosphate, and sulfate limitations, there are 93, 54, and 44 such alleviations of haploinsufficiency, respectively. This represents an alleviation rate of $30 \%$ and $34 \%$ in phosphate- and glucose-limited media, respectively, and $76 \%$ alleviation in sulfate-limited media. GO enrichments for these alleviated gene deletions include cytosolic ribosomal subunit and ubiquinone metabolic process in glucose limitation; retrograde transport, endosome to Golgi, and large ribosomal subunit for phosphate limitation; and the core mediator complex in sulfate limitation (all $P$ values $<0.05$ with a Bonferroni step down correction).

\section{Fitness effects of LOH are condition-specific}

We next looked across environments to determine if hemizygosity caused larger fitness differences between conditions, or if effects were condition-specific. We calculated the variance in fitness values across conditions of 2775 gene deletions present in all six mass competitions (hybrid and purebred deletion collections completed in the three nutrient limitations). Hybrid deletion strains had significantly larger variance between conditions relative to their purebred counterparts $\left(t\right.$-test, $\left.P<2.2 \times 10^{-16}\right)$. In the hybrid genetic context, 92 deletion strains showed antagonistic pleiotropy-low fitness in one condition and high fitness in another. These genes were enriched for GO terms gene expression and RNA metabolic process $\left(P=2.5 \times 10^{-6}\right.$, and $P=4.3 \times 10^{-6}$, Bonferroni corrected).

One consequence of these patterns is that fitness in one nutrient limitation did not predict fitness in the others (Fig. 2). No loci showed consistent fitness differences across all three environments and in both genetic backgrounds. However, 89 deletions were haploinsufficient in two of the media, with no effect in the third (Supplemental Table S5), and 22 genes were haploproficient 


\section{Hybrid Deletion Strains}

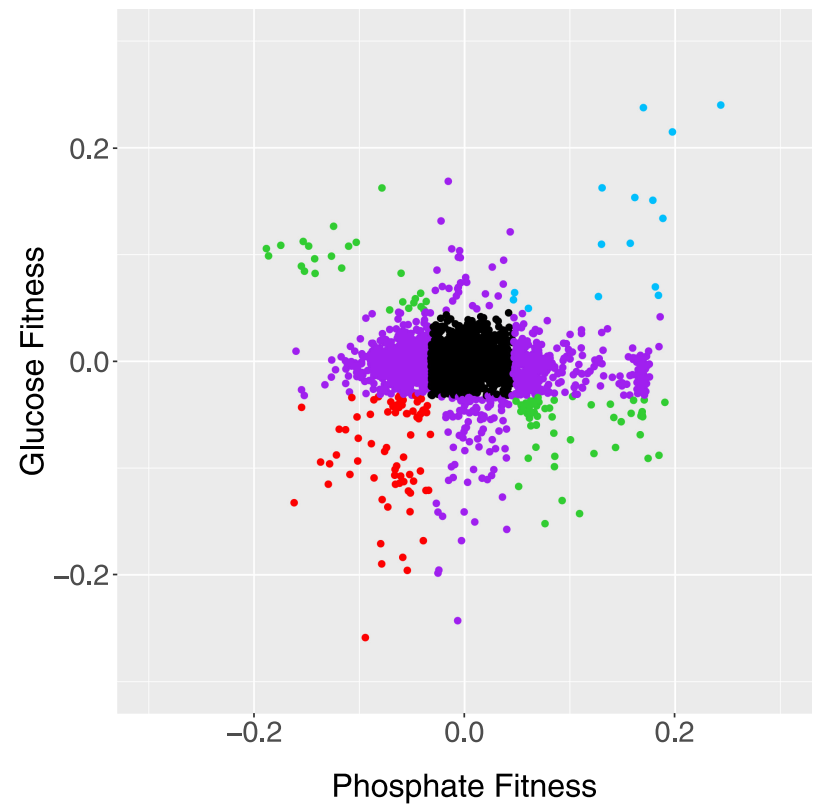

Figure 2. Fitness values of hybrids compared in glucose and phosphate limitation. Strains in black fall inside the $1 \%$ cutoff in both axes, purple strains fall outside the $1 \%$ cutoff in just one axis, and the other colors fall outside of the cutoffs in both axes. Comparisons between other media are shown in Supplemental Figure S5. Data from Supplemental Table S1. $R^{2}=0.00$.

in two media and neutral in the third (Table 2). These genes are of particular interest because they may allow hybrid strains to adapt to multiple or heterogeneous environments. We previously found mutations in one of these genes, MHR1, in two phosphate-limited evolved populations (Smukowski Heil et al. 2017), showing the efficacy of this approach in finding potential driver mutations.

\section{Fitness effects of $\mathrm{LOH}$ are allele-specific}

In our previous work, we found that loss of heterozygosity at the PHO84 locus was beneficial when either allele was lost, i.e., heterozygosity itself had a cost (Smukowski Heil et al. 2017). To determine whether this phenomenon is widespread in our genomescale data set, we performed reciprocal hemizygosity analyses (Steinmetz et al. 2002). We deleted 11 S. uvarum genes that represented a broad range of fitness values (Supplemental Table S6) and mated these strains to WT $S$. cerevisiae, creating a set of reciprocal deletion strains versus our original experiment. We then competed each strain against a WT hybrid labeled with GFP in the indicated nutrient limitation. With one exception (TPK3), the fitness values of these experiments were uncorrelated with those obtained with the corresponding $S$. cerevisiae allele deleted (Supplemental Table S6; Fig. 3). In the gene set tested, S. cerevisiae hemizygous deletions have higher fitness in 13 out of 16 instances versus the matched $S$. uvarum allele deletions. Analysis of additional $S$. uvarum gene deletions will be required to determine whether this bias is meaningful.

\section{Candidate driver genes in $\mathrm{LOH}$ regions recovered in experimental evolution}

Together, these results show that beneficial mutations in hybrids cannot be predicted on the basis of screens performed in S. cerevi- siae alone. LOH events may be tens of kilobases long and include hundreds of genes, making it impractical to use single gene approaches to understand such events. Instead, we used this genome-wide hybrid screen to identify candidate beneficial mutations implicated in LOH events from evolved strains (Smukowski Heil et al. 2017). Six out of 16 hybrid evolved strains contained a total of nine $\mathrm{LOH}$ regions, and four of these regions eliminated the $S$. cerevisiae portion of the genome. These strains have fitness benefits compared to the fully heterozygous ancestor strain, though the relative contribution of each mutation present has not yet been elucidated. One strain containing two LOH regions was evolved in sulfate limitation, where adaptation is largely dominated by amplification of the SUL1 sulfate transporter gene (Brewer et al. 2015; Sanchez et al. 2017). In this strain, we did not identify any single candidate gene deletions that had fitness benefits above our control strain cutoff of 0.046 in either region, consistent with this hypothesis. However, in phosphate limitation, we found candidate driver mutations for the regions deleted in both evolved strains (Table 3). Similar to our results for the whole data set, none of these candidate gene deletions were beneficial in other nutrient limitations in the hybrid context or in any nutrient limitation in the $S$. cerevisiae diploid. They also spanned a diverse variety of biological processes, including a gene of unknown function. The fitness improvements caused by deletion of these candidate driver genes could partly explain the evolved strains' selective benefits, though further follow-up experiments will be required to prove this. We hypothesized that these genes might represent particularly beneficial gene deletions, which could explain the observation of the regions of LOH. However, the candidates were not necessarily among the very top beneficial mutations present over the entire data set. Likewise, adding up the fitness values of genes included in a genome segment does not identify these regions a priori as exceptionally beneficial targets of LOH. Given the small number of evolved strains we have collected in which a $S$. cerevisiae segment is lost, it is unlikely that we have "saturated

Table 2. Condition-dependent haploproficiency in hybrid yeast

\begin{tabular}{llccc}
\hline $\begin{array}{l}\text { Deleted S. cerevisiae } \\
\text { ORF }\end{array}$ & $\begin{array}{c}\text { Gene } \\
\text { symbol }\end{array}$ & Phosphate & Glucose & Sulfate \\
\hline YBL086C & NA & 0.184 & 0.062 & 0.001 \\
YBR123C & TFC1 & 0.181 & 0.07 & 0.016 \\
YDL013W & SLX5 & 0.131 & 0.163 & -0.007 \\
YDR296W & MHR1 & 0.188 & 0.134 & 0.007 \\
YDR325W & YCG1 & 0.037 & 0.095 & 0.094 \\
YDR495C & VPS3 & 0.051 & -0.012 & 0.051 \\
YHR025W & THR1 & 0.056 & 0.002 & 0.049 \\
YJL140W & RPB4 & 0.13 & 0.11 & 0.014 \\
YJL141C & YAK1 & 0.157 & 0.111 & -0.006 \\
YJR064W & CCT5 & 0.128 & 0.002 & 0.049 \\
YJR113C & RSM7 & 0.076 & -0.011 & 0.047 \\
YKR079C & TRZ1 & 0.061 & 0.05 & 0.023 \\
YLL010C & PSR1 & 0.079 & 0.038 & 0.048 \\
YLL027W & ISA1 & 0.047 & 0.064 & -0.011 \\
YLR185W & RPL37A & 0.127 & 0.061 & -0.012 \\
YLR196W & PWP1 & 0.046 & 0.058 & 0.016 \\
YLR280C & NA & 0.055 & 0.045 & 0.059 \\
YML015C & TAF11 & 0.069 & 0.011 & 0.048 \\
YMR142C & RPL13B & 0.179 & 0.151 & -0.02 \\
YOL090W & MSH2 & -0.016 & 0.169 & 0.057 \\
YOL120C & RPL18A & 0.085 & -0.03 & 0.05 \\
YOR001W & RRP6 & 0.056 & -0.015 & 0.048 \\
\hline
\end{tabular}

The last three columns are the fitness values of hemizygous deletion strains grown in the labeled limitation. 


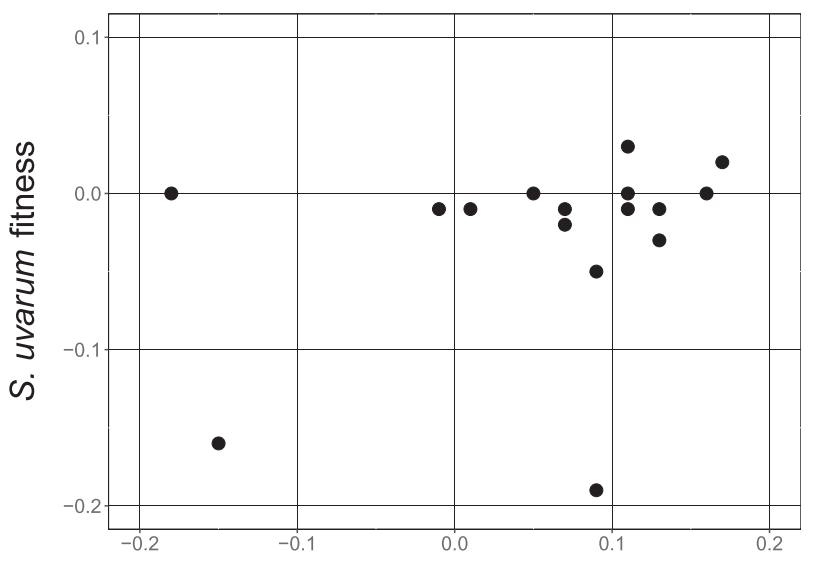

S. cerevisiae fitness

Figure 3. Reciprocal hemizygosity analysis correlation between $S$. cerevisiae deletion ( $x$-axis) and S. uvarum deletion ( $y$-axis). $R^{2}=0.09, P=0.25(P$ value for correlation). All nutrient environments included.

the screen" for beneficial mutations, so these particular events might not, in fact, be expected to be enriched for fitness maxima. Additionally, how best to integrate fitness effects over multiple genes in a region remains uncertain (see Discussion). However, these genes do present attractive targets for further analysis of these particular strains and provide a plausible explanation for their fitness benefits.

\section{Discussion}

$\mathrm{LOH}$ is prevalent in hybrid genomes across taxa. In our previous work, we observed these events arising quickly in interspecific yeast hybrids after a few hundred generations of laboratory selection (Smukowski Heil et al. 2017, 2019). In this study, we found specific gene deletions within these regions that might contribute to the fitness benefits enjoyed by these evolved strains, and we broadened our analysis to the whole genome to determine how hemizygous deletions behave more generally. We find that hybrids are more likely than purebred diploids to benefit from hemizygous deletions (but also to suffer fitness penalties). One possible explanation is alleviation of genetic incompatibilities in the hybrid. However, these benefits are complex-hemizygous deletions are largely condition-, allele-, and species-specific. Our results suggest that $\mathrm{LOH}$ may be an attractive means by which hybrids can adapt to strong, narrow selection pressures but at the potential cost of reduced fitness in alternate environments. Industrial and fermentation environments, where yeast hybrids are successful, might provide exactly such a scenario (Hittinger 2013; Krogerus et al. 2017, 2018).

Specific haploproficient loci rarely overlap between $S$. cerevisiae diploids and interspecific hybrids, indicating that simple gene dosage changes are unlikely to explain their adaptive benefit and/or that dosage sensitivity is strongly dependent on species context. Furthermore, the specific loci are largely private to single selection environments, agreeing with our previous experimental evolution findings showing repeated occurrence of $\mathrm{LOH}$ for specific regions was also condition-specific. Finally, fitness effects are allele-specific-fitness consequences of deletion of the $S$. cerevisiae allele in the hybrid had almost no correlation with the fitness consequences of deletion of the $S$. uvarum allele. Again, these results are consistent with prior observations of species preference for LOH in evolved hybrids. However, we acknowledge our relatively small sample size, and a larger study targeted at this problem might yield a more nuanced view. Nonetheless, these results argue that, if relief of genetic incompatibilities is a relevant mechanistic explanation for adaptation, then such incompatibilities must be acting in an allele-specific manner.

Though we have focused here on beneficial gene deletions, we note that other groups have generated similar data sets to look at fitness changes in both directions in hybrids carrying single allele deletions or transposon insertions (Herbst et al. 2017; Weiss et al. 2018). In particular, we largely confirm the gross patterns observed in a collection of $S$. paradoxus $\times S$. cerevisiae hybrids deleted for $S$. cerevisiae alleles. However, we see no correlations across studies in the types of genes found to exhibit these more extreme fitness effects. Given the different conditions and different species used, and since both sets of results found dependencies on medium and genetic background, it is not clear to what degree we should expect to see overlap. A better controlled study where both strain collections are tested in parallel in the same conditions would be informative along these lines.

We note several additional points for improvement and further study. First, while we did observe some significant GO enrichments among gene deletions with shared fitness characteristics (for example, differences in ribosomal structure/function and expression differences may contribute to differential fitness in various genetic and environmental contexts, respectively), the lack of strong biological process enrichments provided no simple rationale for the molecular explanations underlying these effects. Combining our data with other results collected from hybrids, such as protein-protein interactions (Chrétien et al. 2018), may help provide such explanations. Expanding our study to additional genes would also be desirable. Due to the method we used to generate the hybrid deletion strains, we were limited to genes that are nonessential in $S$. cerevisiae. Though we deleted a small number of $S$. uvarum genes to compare with the orthologous $S$. cerevisiae allele deletions, we did not explore $S$. uvarum genes genomewide. We hope to remedy this in the future using our recently created S. uvarum insertional mutagenesis library (Sanchez et al. 2019). In vivo transposition is another potential approach that has recently been applied to $S$. paradoxus $\times S$. cerevisiae hybrids (Weiss et al. 2018). Both these approaches have some advantages over the $S$. cerevisiae deletion collection, which has potential

Table 3. Candidate driver genes in deleted S. cerevisiae genomic segments in evolved phosphate-limited hybrid strains

\begin{tabular}{lcc}
\hline $\begin{array}{l}\text { Candidate driver mutation } \\
\text { (systematic gene name of } \\
\text { S. cerevisiae deleted gene) }\end{array}$ & $\begin{array}{c}\text { Gene } \\
\text { symbol }\end{array}$ & $\begin{array}{c}\text { Competitive fitness in } \\
\text { phosphate limitation from } \\
\text { hemizygous hybrid screen }\end{array}$ \\
\hline YIR023W & DAL81 & 0.132 \\
YML041C & VPS71 & 0.080 \\
YML049C & RSE1 & 0.171 \\
YML061C & PIF1 & 0.048 \\
YML066C & SMA2 & 0.072 \\
YML069W & POB3 & 0.055 \\
YML091C & RPM2 & 0.067 \\
YML105C & SEC65 & 0.068 \\
YML112W & CTK3 & 0.169 \\
YML115C & VAN1 & 0.168 \\
YML128C & MSC1 & 0.131 \\
YML130C & ERO1 & 0.061 \\
YML131W & NA & 0.051 \\
\hline
\end{tabular}


problems with suppressor mutations, mutation accumulation, and aneuploidy (Hughes et al. 2000; Teng et al. 2013; van Leeuwen et al. 2016).

Finally, we have not been able to determine how multiple genes within a segment combine to generate their full fitness consequence, a topic that has bedeviled the aneuploidy field more broadly (Solimini et al. 2012; Davoli et al. 2013; Sunshine et al. 2015; Dodgson et al. 2016; Iyer et al. 2018). Comparison of the fitness values of the evolved hybrids with the fitness of single gene deletions revealed cases where a simple additive model both under- or overestimated the evolved strain fitness (Supplemental Table S7). However, the evolved strains are an imperfect basis for comparison since they contain other mutations in addition to the hemizygous region. Alternative selection methods for recovering high-fitness strains with a minimal number of additional mutations presents one possible approach (Bellon et al. 2018). An even better approach might be to create hybrids with segmental monosomy and test their fitness directly. Such strains could be engineered using chromosome fragmentation vectors or recombinase-based approaches such as the SCRaMbLE system (Morrow et al. 1997; Dymond et al. 2011; Shen et al. 2018; Li et al. 2019).

\section{Methods}

\section{Strains and collections}

The $S$. cerevisiae strain collections are described in Payen et al. (2016).

All $S$. uvarum strains are derived from the reference strain CBS 7001, sometimes identified as $S$. bayanus var uvarum.

Hybrid collections were made by spreading $200 \mu \mathrm{L}$ mid-log $S$. uvarum lys2 MAT $\alpha$ on solid YPD omni plates, then spotting the appropriate haploid MATa collection (deletion or barcoder) using a pinner with 96 arrayed pins. After overnight growth, colonies were transferred to selective solid minimal media to ensure only hybrid growth. These plates were then transferred to liquid selective media and pooled.

S. uvarum deletion strains were provided by Sarah Bissonnette and Jasper Rine (UC Berkeley) (Sanchez et al. 2019).

\section{Fitness assays and barcode sequencing}

All collections were grown as pools in duplicate in three different chemostat media-glucose-limited, phosphate-limited, and sulfate-limited. All S. cerevisiae competition experiments were taken from Payen et al. (2016), where the protocol is described in detail. Briefly, pools were inoculated into $240-\mathrm{mL}$ chemostats and grown for $24 \mathrm{~h}$, when peristaltic pumps were turned on at a dilution rate of $\sim 0.17$ volumes per hour (Payen et al. 2016). Samples were taken from chemostats twice a day. From these samples, the unique DNA barcodes from each collection were PCR-amplified, with each time point having a unique Illumina adapter incorporated during PCR amplification. The barcodes were then sequenced on an Illumina Genome Analyzer IIx. Data analysis was performed as described below, and all analysis scripts are provided as Supplemental Code. The frequency of the barcodes was used to calculate the fitness of each strain by determining the natural log in the change of proportional barcode frequency over 25 generations, which is the longest competitions can go without seeing selection from de novo mutations. We required a minimum of 100 barcode counts per strain to be identified. For the reciprocal hemizygosity analysis, the 100-barcode limit was reduced to 44 for comparison to the mass competitions because manual curating of these ensured no false positives. The number 44 was chosen because it corresponded with S. uvarum strains we possessed, and it was sufficiently high to detect a trend. Many sequences for the WT barcoded collection DNA barcodes were only determinable through examination of overrepresented sequences in sequencing data, and these were used for analysis.

Treatment of the data for each of the en masse experiments was as follows. Basic QC was performed on the data, eliminating reads with poor quality (Payen et al. 2016). The raw reads for every barcode/strain were recorded for every time point, using only exact matches for the barcode. A second table was then made to normalize to total reads at each time point by dividing each strain by the total identified barcodes for every time. A third table was then made to find the relative abundance of each strain at each time point by dividing each value by generation 0 . If the total raw reads were not more than 100 at this point, that strain was removed from analyses. A fourth table was made from the natural log of the third table. Lastly, linear regression was performed on each strain in the fourth table with the time in generations. This slope is the relative fitness.

Individual competition experiments were done in the respective media in 20-mL chemostats and competed against a single WT clone with a GFP label. Relative strain abundance was monitored using a BD Accuri C6 flow cytometer. Fitness was determined by regressing the slope of generations versus the $\ln$ (dark cells/GFP cells).

\section{GO enrichments in the data set}

GO enrichments were determined using the ClueGO application in Cytoscape (Bindea et al. 2009) and using the total strains identified in our experiments as the background population. Outliers were determined using a $1 \%$ cutoff in each direction based on the WT barcoded collection. All ontologies were corrected for multiple comparisons with a Bonferroni step down analysis.

\section{Statistics}

Statistical measures, unless otherwise stated, were performed in $\mathrm{R}$ (R Core Team 2017). The statistics used are stated in the results adjacent to $P$-values. To assess comparison of variance, each en masse experiment was rank-ordered to eliminate differences between conditions or genetic backgrounds. Only the 2775 strains in all six experiments were included. The variance was determined for each strain in the hybrid and purebreds across the three conditions. This generated 2775 variance measures for the hybrids and the same number for the purebreds. To test if these variances were significantly different, a $t$-test was performed between the purebred and hybrid variances.

\section{Data access}

The sequencing data generated in this study have been submitted to the NCBI BioProject database (https://www.ncbi.nlm.nih.gov/ bioproject/) under accession number PRJNA283983. All scripts generated in this study are available as Supplemental Code.

\section{Acknowledgments}

We thank Caitlin Connelly for her contributions to the initiation of this project. We also thank Corey Nislow, Jasper Rine, and Sarah Bissonnette for sharing strains. This work was funded by National Science Foundation grant 1516330 and by P41 GM103533 from the National Institute of General Medical Sciences from the National Institutes of Health. S.M.L. was funded by T32 HG00035 from the National Human Genome Research Institute

\section{Genome Research}

www.genome.org 
from the National Institutes of Health. M.J.D. is a Senior Fellow in the Genetic Networks program at the Canadian Institute for Advanced Research. The research of M.J.D. was supported in part by a Faculty Scholar grant from the Howard Hughes Medical Institute.

\section{References}

Almeida P, Goncalves C, Teixeira S, Libkind D, Bontrager M, MasneufPomarède I, Albertin W, Durrens P, Sherman DJ, Marullo P, et al. 2014. A Gondwanan imprint on global diversity and domestication of wine and cider yeast Saccharomyces uvarum. Nat Commun 5: 4044. doi:10.1038/ncomms5044

Bellon JR, Ford CM, Borneman AR, Chambers PJ. 2018. A novel approach to isolating improved industrial interspecific wine yeasts using chromosomal mutations as potential markers for increased fitness. Front Microbiol 9: 1442. doi:10.3389/fmicb.2018.01442

Bindea G, Mlecnik B, Hackl H, Charoentong P, Tosolini M, Kirilovsky A, Fridman WH, Pagès F, Trajanoski Z, Galon J. 2009. ClueGO: a Cytoscape plug-in to decipher functionally grouped gene ontology and pathway annotation networks. Bioinformatics 25: 1091-1093. doi:10.1093/bioinformatics/btp101

Borneman AR, Zeppel R, Chambers PJ, Curtin CD. 2014. Insights into the Dekkera bruxellensis genomic landscape: Comparative genomics reveals variations in ploidy and nutrient utilisation potential amongst wine isolates. PLoS Genet 10: e1004161. doi:10.1371/journal.pgen.1004161

Brenchley R, Spannagl M, Pfeifer M, Barker GL, D'Amore R, Allen AM, McKenzie N, Kramer M, Kerhornou A, Bolser D, et al. 2012. Analysis of the bread wheat genome using whole-genome shotgun sequencing. Nature 491: 705-710. doi:10.1038/nature 11650

Brewer BJ, Payen C, Di Rienzi SC, Higgins MM, Ong G, Dunham MJ, Raghuraman MK. 2015. Origin-dependent inverted-repeat amplification: tests of a model for inverted DNA amplification. PLoS Genet 11: e1005699. doi:10.1371/journal.pgen.1005699

Chen J, Upadhyaya NM, Ortiz D, Sperschneider J, Li F, Bouton C, Breen S, Dong C, Xu B, Zhang X, et al. 2017. Loss of AvrSr50 by somatic exchange in stem rust leads to virulence for $S r 50$ resistance in wheat. Science 358: 1607-1610. doi:10.1126/science.aao4810

Chester M, Leitch AR, Soltis PS, Soltis DE. 2010. Review of the application of modern cytogenetic methods (FISH/GISH) to the study of reticulation (polyploidy/hybridisation). Genes (Basel) 1: 166-192. doi:10.3390/ genes1020166

Chrétien AE, Gagnon-Arsenault I, Dubé AK, Barbeau X, Després PC, Lamothe C, Dion-Côté AM, Lagüe P, Landry CR. 2018. Extended linkers improve the detection of protein-protein interactions (PPIs) by dihydrofolate reductase protein-fragment complementation assay (DHFR PCA) in living cells. Mol Cell Proteomics 17: 549. doi:10.1074/mcp.A117 .000385

Csoma H, Zakany N, Capece A, Romano P, Sipiczki M. 2010. Biological diversity of Saccharomyces yeasts of spontaneously fermenting wines in four wine regions: comparative genotypic and phenotypic analysis. Int J Food Microbiol 140: 239-248. doi:10.1016/j.ijfoodmicro.2010.03 .024

Dannemann M, Andrés AM, Kelso J. 2016. Introgression of Neandertal- and Denisovan-like haplotypes contributes to adaptive variation in human toll-like receptors. Am J Hum Genet 98: 22-33. doi:10.1016/j.ajhg.2015 .11 .015

Davoli T, Xu AW, Mengwasser KE, Sack LM, Yoon JC, Park PJ, Elledge SJ. 2013. Cumulative haploinsufficiency and triplosensitivity drive aneuploidy patterns and shape the cancer genome. Cell 155: 948-962. doi:10.1016/i.cell.2013.10.011

Delneri D, Hoyle DC, Gkargkas K, Cross EJ, Rash B, Zeef L, Leong HS, Davey HM, Hayes A, Kell DB, et al. 2008. Identification and characterization of high-flux-control genes of yeast through competition analyses in continuous cultures. Nat Genet 40: 113-117. doi:10.1038/ng.2007.49

Deutschbauer AM, Davis RW. 2005. Quantitative trait loci mapped to single-nucleotide resolution in yeast. Nat Genet 37: 1333-1340. doi:10 $.1038 / \mathrm{ng} 1674$

Dodgson SE, Kim S, Costanzo M, Baryshnikova A, Morse DL, Kaiser CA, Boone C, Amon A. 2016. Chromosome-specific and global effects of aneuploidy in Saccharomyces cerevisiae. Genetics 202: 1395-1409. doi:10 $.1534 /$ genetics. 115.185660

Dunn B, Sherlock G. 2008. Reconstruction of the genome origins and evolution of the hybrid lager yeast Saccharomyces pastorianus. Genome Res 18: 1610-1623. doi:10.1101/gr.076075.108

Dymond JS, Richardson SM, Coombes CE, Babatz T, Muller H, Annaluru N, Blake WJ, Schwerzmann JW, Dai J, Lindstrom DL, et al. 2011. Synthetic chromosome arms function in yeast and generate phenotypic diversity by design. Nature 477: 471-476. doi:10.1038/nature10403
Emery M, Willis MMS, Hao Y, Barry K, Oakgrove K, Peng Y, Schmutz J, Lyons E, Pires JC, Edger PP, et al. 2018. Preferential retention of genes from one parental genome after polyploidy illustrates the nature and scope of the genomic conflicts induced by hybridization. PLoS Genet 14: e1007267. doi:10.1371/journal.pgen.1007267

Giaever G, Chu AM, Ni L, Connelly C, Riles L, Veronneau S, Dow S, LucauDanila A, Anderson K, Andre B, et al. 2002. Functional profiling of the Saccharomyces cerevisiae genome. Nature 418: 387-391. doi:10.1038/ nature00935

Gittelman RM, Schraiber JG, Vernot B, Mikacenic C, Wurfel MM, Akey JM. 2016. Archaic hominin admixture facilitated adaptation to out-of-Africa environments. Curr Biol 26: 3375-3382. doi:10.1016/j.cub.2016.10.041

Gonzalez SS, Barrio E, Gafner J, Querol A. 2006. Natural hybrids from Saccharomyces cerevisiae, Saccharomyces bayanus and Saccharomyces kudriavzevii in wine fermentations. FEMS Yeast Res 6: 1221-1234. doi:10.1111/j.1567-1364.2006.00126.x

Gresham D, Desai MM, Tucker CM, Jenq HT, Pai DA, Ward A, DeSevo CG Botstein D, Dunham MJ. 2008. The repertoire and dynamics of evolutionary adaptations to controlled nutrient-limited environments in yeast. PLoS Genet 4: e1000303. doi:10.1371/journal.pgen.1000303

Herbst RH, Bar-Zvi D, Reikhav S, Soifer I, Breker M, Jona G, Shimoni E, Schuldiner M, Levy AA, Barkai N. 2017. Heterosis as a consequence of regulatory incompatibility. BMC Biol 15: 38. doi:10.1186/s12915-0170373-7

Hittinger CT. 2013. Saccharomyces diversity and evolution: a budding model genus. Trends Genet 29: 309-317. doi:10.1016/j.tig.2013.01.002

Huerta-Sánchez E, Jin X, Asan, Bianba Z, Peter BM, Vinckenbosch N, Liang Y, Yi X, He M, Somel M, et al. 2014. Altitude adaptation in Tibetans caused by introgression of Denisovan-like DNA. Nature 512: 194-197. doi:10.1038/nature13408

Hughes TR, Roberts CJ, Dai H, Jones AR, Meyer MR, Slade D, Burchard J, Dow S, Ward TR, Kidd MJ, et al. 2000. Widespread aneuploidy revealed by DNA microarray expression profiling. Nat Genet 25: 333-337. doi:10 $1038 / 77116$

Iyer J, Singh MD, Jensen M, Patel P, Pizzo L, Huber E, Koerselman H, Weiner AT, Lepanto P, Vadodaria K, et al. 2018. Pervasive genetic interactions modulate neurodevelopmental defects of the autism-associated 16p11.2 deletion in Drosophila melanogaster. Nat Commun 9: 2548. doi:10.1038/s41467-018-04882-6

Krogerus K, Magalhães F, Vidgren V, Gibson B. 2017. Novel brewing yeast hybrids: creation and application. Appl Microbiol Biotechnol 101: 6578. doi:10.1007/s00253-016-8007-5

Krogerus K, Holmstrom S, Gibson B. 2018. Enhanced wort fermentation with de novo lager hybrids adapted to high-ethanol environments. Appl Environ Microbiol 84: e02302-17. doi:10.1128/AEM.02302-17

Kunicka-Styczyńska A, Rajkowska K. 2011. Physiological and genetic stability of hybrids of industrial wine yeasts Saccharomyces sensu stricto complex. J Appl Microbiol 110: 1538-1549. doi:10.1111/j.1365-2672.2011 .05009.X

Li Y, Wu Y, Ma L, Guo Z, Xiao W, Yuan Y. 2019. Loss of heterozygosity by SCRaMbLEing. Sci China Life Sci 62: 381-393. doi:10.1007/s11427019-9504-5

Libkind D, Hittinger CT, Valerio E, Goncalves C, Dover J, Johnston M, Goncalves P, Sampaio JP. 2011. Microbe domestication and the identification of the wild genetic stock of lager-brewing yeast. Proc Natl Acad Sci 108: 14539-14544. doi:10.1073/pnas.1105430108

Louis VL, Despons L, Friedrich A, Martin T, Durrens P, Casarégola S, Neuvéglise C, Fairhead C, Marck C, Cruz JA, et al. 2012. Pichia sorbitophila, an interspecies yeast hybrid, reveals early steps of genome resolution after polyploidization. G3 (Bethesda) 2: 299-311. doi:10.1534/g3 .111 .000745

Maclean CJ, Greig D. 2008. Prezygotic reproductive isolation between Saccharomyces cerevisiae and Saccharomyces paradoxus. BMC Evol Biol 8: 1. doi:10.1186/1471-2148-8-1

Mallet J. 2005. Hybridization as an invasion of the genome. Trends Ecol Evol 20: 229-237. doi:10.1016/j.tree.2005.02.010

Marcet-Houben M, Gabaldón T. 2015. Beyond the whole-genome duplication: phylogenetic evidence for an ancient interspecies hybridization in the baker's yeast lineage. PLoS Biol 13: e1002220. doi:10.1371/journal .pbio. 1002220

Martini AV, Martini A. 1987. Three newly delimited species of Saccharomyces sensu stricto. Antonie Van Leeuwenhoek 53: 77-84. doi:10.1007/ BF00419503

Mertens S, Steensels J, Saels V, De Rouck G, Aerts G, Verstrepen KJ. 2015. A large set of newly created interspecific Saccharomyces hybrids increases aromatic diversity in lager beers. Appl Environ Microbiol 81: 82028214. doi:10.1128/AEM.02464-15

Mixão V, Gabaldón T. 2018. Hybridization and emergence of virulence in opportunistic human yeast pathogens. Yeast 35: 5-20. doi:10.1002/ yea.3242 
Morales L, Dujon B. 2012. Evolutionary role of interspecies hybridization and genetic exchanges in yeasts. Microbiol Mol Biol Rev 76: 721-739. doi:10.1128/MMBR.00022-12

Morrow DM, Connelly C, Hieter P. 1997. "Break copy" duplication: a model for chromosome fragment formation in Saccharomyces cerevisiae. Genetics 147: 371-382.

Muller LA, McCusker JH. 2009. A multispecies-based taxonomic microarray reveals interspecies hybridization and introgression in Saccharomyces cerevisiae. FEMS Yeast Res 9: 143-152. doi:10.1111/j.1567-1364.2008 .00464.x

Murphy HA, Zeyl CW. 2012. Prezygotic isolation between Saccharomyces cerevisiae and Saccharomyces paradoxus through differences in mating speed and germination timing. Evolution 66: 1196-1209. doi:10.1111/j.15585646.2011.01516.x

Naumova ES, Naumov GI, Masneuf-Pomarède I, Aigle M, Dubourdieu D. 2005. Molecular genetic study of introgression between Saccharomyces bayanus and S. cerevisiae. Yeast 22: 1099-1115. doi:10.1002/yea.1298

Nguyen HV, Legras JL, Neuvéglise C, Gaillardin C. 2011. Deciphering the hybridisation history leading to the Lager lineage based on the mosaic genomes of Saccharomyces bayanus strains NBRC1948 and CBS380. PLoS One 6: e25821. doi:10.1371/journal.pone.0025821

Ohnuki S, Ohya Y. 2018. High-dimensional single-cell phenotyping reveals extensive haploinsufficiency. PLoS Biol 16: e2005130. doi:10.1371/jour nal.pbio. 2005130

Otto SP, Whitton J. 2000. Polyploid incidence and evolution. Annu Rev Genet 34: 401-437. doi:10.1146/annurev.genet.34.1.401

Payen C, Sunshine AB, Ong GT, Pogachar JL, Zhao W, Dunham MJ. 2016 High-throughput identification of adaptive mutations in experimentally evolved yeast populations. PLoS Genet 12: e1006339. doi:10.1371/ journal.pgen.1006339

Pérez-Través L, Lopes CA, Querol A, Barrio E. 2014. On the complexity of the Saccharomyces bayanus taxon: hybridization and potential hybrid speciation. PLoS One 9: e93729. doi:10.1371/journal.pone.0093729

Peris D, Lopes CA, Belloch C, Querol A, Barrio E. 2012. Comparative genomics among Saccharomyces cerevisiae $\times$ Saccharomyces kudriavzevii natural hybrid strains isolated from wine and beer reveals different origins. $B M C$ Genomics 13: 407. doi:10.1186/1471-2164-13-407

Peris D, Moriarty RV, Alexander WG, Baker E, Sylvester K, Sardi M, Langdon QK, Libkind D, Wang QM, Bai FY, et al. 2017. Hybridization and adaptive evolution of diverse Saccharomyces species for cellulosic biofuel production. Biotechnol Biofuels 10: 78. doi:10.1186/s13068-017-0763-7

Piotrowski JS, Nagarajan S, Kroll E, Stanbery A, Chiotti KE, Kruckeberg AL, Dunn B, Sherlock G, Rosenzweig F. 2012. Different selective pressures lead to different genomic outcomes as newly-formed hybrid yeasts evolve. BMC Evol Biol 12: 46. doi:10.1186/1471-2148-12-46

Pir P, Gutteridge A, Wu J, Rash B, Kell DB, Zhang N, Oliver SG. 2012. The genetic control of growth rate: a systems biology study in yeast. $B M C$ Syst Biol 6: 4. doi:10.1186/1752-0509-6-4

Pryszcz LP, Németh T, Saus E, Ksiezopolska E, Hegedû́sová E, Nosek J, Wolfe KH, Gacser A, Gabaldón T. 2015. The genomic aftermath of hybridization in the opportunistic pathogen Candida metapsilosis. PLoS Genet 11: e1005626. doi:10.1371/journal.pgen.1005626

R Core Team. 2017. R: a language and environment for statistical computing. $\mathrm{R}$ Foundation for Statistical Computing, Vienna. https://www.R-project .org/.

Racimo F, Gokhman D, Fumagalli M, Ko A, Hansen T, Moltke I, Albrechtsen A, Carmel L, Huerta-Sanchez E, Nielsen R. 2017. Archaic adaptive introgression in TBX15/WARS2. Mol Biol Evol 34: 509-524. doi:10.1093/mol $\mathrm{bev} / \mathrm{msw} 283$

Sanchez MR, Miller AW, Liachko I, Sunshine AB, Lynch B, Huang M, Alcantara E, DeSevo CG, Pai DA, Tucker CM, et al. 2017. Differential paralog divergence modulates genome evolution across yeast species. PLoS Genet 13: e1006585. doi:10.1371/journal.pgen.1006585

Sanchez MR, Payen C, Cheong F, Hovde B, Bissonnette S, Arkin A, Skerker JM, Brem R, Caudy AA, Dunham MJ. 2019. Transposon insertional mutagenesis in Saccharomyces uvarum reveals trans-acting effects influenc- ing species-dependent essential genes. Genome Res 29: 396-406. doi: 10.1101/gr.232330.117

Scannell DR, Frank AC, Conant GC, Byrne KP, Woolfit M, Wolfe KH. 2007. Independent sorting-out of thousands of duplicated gene pairs in two yeast species descended from a whole-genome duplication. Proc Natl Acad Sci 104: 8397-8402. doi:10.1073/pnas.0608218104

Schröder MS, Martinez de San Vicente K, Prandini TH, Hammel S, Higgins DG, Bagagli E, Wolfe KH, Butler G. 2016. Multiple origins of the pathogenic yeast Candida orthopsilosis by separate hybridizations between two parental species. PLoS Genet 12: e1006404. doi:10.1371/journal.pgen .1006404

Shen MJ, Wu Y, Yang K, Li Y, Xu H, Zhang H, Li BZ, Li X, Xiao WH, Zhou X, et al. 2018. Heterozygous diploid and interspecies SCRaMbLEing. Nat Commun 9: 1934. doi:10.1038/s41467-018-04157-0

Sipiczki M. 2008. Interspecies hybridization and recombination in Saccharomyces wine yeasts. FEMS Yeast Res 8: 996-1007. doi:10.1111/ .1567-1364.2008.00369.x

Smukowski Heil CS, DeSevo CG, Pai DA, Tucker CM, Hoang ML, Dunham MJ. 2017. Loss of heterozygosity drives adaptation in hybrid yeast. Mol Biol Evol 34: 1596-1612. doi:10.1093/molbev/msx098

Smukowski Heil C, Burton JN, Liachko I, Friedrich A, Hanson NA, Morris CL Schacherer J, Shendure J, Thomas JH, Dunham MJ. 2018. Identification of a novel interspecific hybrid yeast from a metagenomic spontaneously inoculated beer sample using Hi-C. Yeast 35: 71-84. doi:10.1002/yea 3280

Smukowski Heil CS, Large CRL, Patterson K, Hickey AS, Yeh CC, Dunham MJ. 2019. Temperature preference can bias parental genome retention during hybrid evolution. PLoS Genet 15: e1008383. doi:10.1371/jour nal.pgen.1008383

Solimini NL, Xu Q, Mermel CH, Liang AC, Schlabach MR, Luo J, Burrows AE, Anselmo AN, Bredemeyer AL, Li MZ, et al. 2012. Recurrent hemizygous deletions in cancers may optimize proliferative potential. Science 337: 104-109. doi:10.1126/science. 1219580

Steinmetz LM, Sinha H, Richards DR, Spiegelman JI, Oefner PJ, McCusker JH, Davis RW. 2002. Dissecting the architecture of a quantitative trait locus in yeast. Nature 416: 326-330. doi:10.1038/416326a

Stelkens RB, Brockhurst MA, Hurst GD, Miller EL, Greig D. 2014. The effect of hybrid transgression on environmental tolerance in experimental yeast crosses. J Evol Biol 27: 2507-2519. doi:10.1111/jeb.12494

Sun S, Xu J. 2009. Chromosomal rearrangements between serotype A and D strains in Cryptococcus neoformans. PLoS One 4: e5524. doi:10.1371/jour nal.pone.0005524

Sunshine AB, Payen C, Ong GT, Liachko I, Tan KM, Dunham MJ. 2015. The fitness consequences of aneuploidy are driven by condition-dependent gene effects. PLoS Biol 13: e1002155. doi:10.1371/journal.pbio .1002155

Teng X, Dayhoff-Brannigan M, Cheng WC, Gilbert CE, Sing CN, Diny NL, Wheelan SJ, Dunham MJ, Boeke JD, Pineda FJ, et al. 2013. Genome-wide consequences of deleting any single gene. Mol Cell 52: 485-494. doi:10 .1016/j.molcel.2013.09.026

van Leeuwen J, Pons C, Mellor JC, Yamaguchi TN, Friesen H, Koschwanez J, Usaj MM, Pechlaner M, Takar M, Usaj M, et al. 2016. Exploring genetic suppression interactions on a global scale. Science 354: aag0839. doi:10 $.1126 /$ science.aag0839

Weiss CV, Roop JI, Hackley RK, Chuong JN, Grigoriev IV, Arkin AP, Skerker JM, Brem RB. 2018. Genetic dissection of interspecific differences in yeast thermotolerance. Nat Genet 50: 1501-1504. doi:10.1038/s41588018-0243-4.

Yan Z, Costanzo M, Heisler LE, Paw J, Kaper F, Andrews BJ, Boone C, Giaever G, Nislow C. 2008. Yeast Barcoders: a chemogenomic application of a universal donor-strain collection carrying bar-code identifiers. Nat Methods 5: 719-725. doi:10.1038/nmeth.1231

Received October 24, 2018; accepted in revised form August 7, 2019. 


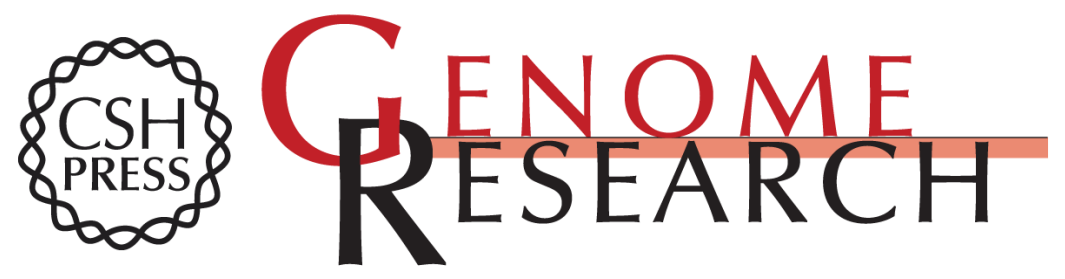

\section{Fitness benefits of loss of heterozygosity in Saccharomyces hybrids}

Samuel M. Lancaster, Celia Payen, Caiti Smukowski Heil, et al.

Genome Res. 2019 29: 1685-1692 originally published online September 23, 2019

Access the most recent version at doi:10.1101/gr.245605.118

Supplemental Material

References

Creative

Commons

License

Email Alerting

Service
http://genome.cshlp.org/content/suppl/2019/09/23/gr.245605.118.DC1

This article cites 73 articles, 13 of which can be accessed free at: http://genome.cshlp.org/content/29/10/1685.full.html\#ref-list-1

This article is distributed exclusively by Cold Spring Harbor Laboratory Press for the first six months after the full-issue publication date (see

$\mathrm{http}: / / g$ enome.cshlp.org/site/misc/terms.xhtml). After six months, it is available under a Creative Commons License (Attribution-NonCommercial 4.0 International), as described at http://creativecommons.org/licenses/by-nc/4.0/.

Receive free email alerts when new articles cite this article - sign up in the box at the top right corner of the article or click here.

\section{Affordable, Accurate Sequencing.}

To subscribe to Genome Research go to:

https://genome.cshlp.org/subscriptions 